\title{
Assessment of the effects of prostaglandins on myometrial and leiomyoma cells in vitro through microRNA profiling
}

\author{
HWI GON KIM ${ }^{1,2}$, GA YOUNG JUNG ${ }^{3}$, SEUNG BIN PARK ${ }^{3}$, YEON JEAN CHO ${ }^{3}$ and MYOUNGSEOK HAN ${ }^{3}$ \\ ${ }^{1}$ Department of Obstetrics and Gynecology; ${ }^{2}$ Research Institute for Convergence of Biomedical Science and Technology, \\ Pusan National University Yangsan Hospital, Yangsan, Gyeongsangnam 50612; ${ }^{3}$ Department of Obstetrics and Gynecology, \\ Dong-A University, College of Medicine, Busan 49201, Republic of Korea
}

Received November 22, 2017; Accepted June 11, 2018

DOI: $10.3892 / \mathrm{mmr} .2018 .9160$

\begin{abstract}
It is well known that prostaglandin (PG) E2 and PGF $2 \alpha$ are secreted in copious amounts from the menstruating uterus. The aim of the present study was to determine whether PGs affect the growth of uterine leiomyomas (ULs) to the same extent as estrogen or progesterone (P4). The present study evaluated the expression of eight microRNAs (miRNAs) by reverse transcription-quantitative polymerase chain reaction (RT-qPCR) through treatment with estradiol (E2), P4, PGE2, PGF $2 \alpha$ and each antagonist or cyclooxygenase-2 (COX-2) inhibitor of cultured leiomyoma and myometrial cells (LC and $\mathrm{MC}$, respectively). The eight miRNAs were divided into two groups according to their primary biological action, namely apoptosis-regulating miRNAs (let-7a, miR-21, miR-26a and miR-200a) and inflammation-regulating miRNAs (miR-29b, miR-93, miR-106b and miR-100b). PGE2 induced significantly higher expression of the 3 anti-apoptotic miRs, let-7a, miR-16a and miR-200a, in LC when compared with the non-treated control or E2. PGE2 significantly promoted a greater expression of let-7a and miR-26a in LC when compared with P4. Overall, PGE2 exerted the highest anti-apoptotic and anti-inflammatory effect in LC, which was comparable with E2. It was not observed among the inflammation-regulating miRNAs in LC. PGF $2 \alpha$ did not exert effects as prominent as those of PGE2. In MC, PGs and sex steroids exerted no similar effects on MC compared with LC. The present study demonstrated that PGE2 levels during menstruation may affect the growth of preexisting ULs without affecting the normal myometrium. Therefore, the control of secretion of PGs from the menstruating uterus or the administration of antagonists may be an alternative therapy for inhibiting the growth of ULs.
\end{abstract}

Correspondence to: Professor Myoungseok Han, Department of Obstetrics and Gynecology, Dong-A University, College of Medicine, Daesingongwon-Ro 32 (Dongdaesin-Dong 3), Seo-Gu, Busan 49201, Republic of Korea

E-mail: hmsobgy@dau.ac.kr

Key words: leiomyoma, myometrium, prostaglandin, microRNA, estrogen, progesterone

\section{Introduction}

Uterine leiomyomas (ULs) are the most common tumors of the female reproductive organs, occurring in $\leq 80 \%$ of all women of reproductive age, with $\leq 30 \%$ of women complaining of severe symptoms and seeking treatment $(1,2)$. ULs are associated with a range of symptoms, including pressure-associated symptoms, uterine bleeding, dysmenorrhea and infertility. It is well known that the growth of ULs is primarily dependent on stimulation by sex steroid hormones, estrogen (E) and progesterone (P4), secreted by the ovary. However, it is not clear whether prostaglandins (PGs), including PGE2 and PGF2 $\alpha$, which are produced in large amounts by the menstruating uterus, affect the growth of ULs to the same extent as E or P4.

PGs are generated by almost every tissue in the body and act as important messengers or effectors in a wide variety of functions, particularly in the inflammatory response. Their biosynthesis is significantly increased in inflamed tissues, such as the menstruating uterus. Although a large amount of PGs are produced in the uterus during the menstrual period, the precise roles of PGs have not been extensively investigated (2). In a previous study, it was demonstrated that the cyclooxygenase-2 (COX-2) inhibitor celecoxib inhibited the proliferation of ULs by inhibiting the $\mathrm{NF}-\kappa \mathrm{B}$ pathway in cultured leiomyoma cells (3). The study indicated that PGs from the uterus may affect proliferation through intracellular signaling pathways to some extent. Therefore, PGs may not only serve a key role in the generation of the inflammatory response, but they may also affect the intracellular processes promoting tumor growth. However, that study did not elucidate whether PGs induced cell proliferation or inflammation to the same extent as ovarian sex steroids, namely E and P4. Although PGE2 is one of the most abundant PGs in the body, is widely described in animal species and exhibits useful biological activities, it has not been clearly determined whether it is able to stimulate UL growth.

MicroRNAs (miRNAs) are a novel class of regulators that have been demonstrated to downregulate gene expression by blocking mRNA translation and/or degrading the mRNA transcript, depending on the level of complementarity between the miRNA and its target (4). miRNAs are essential for normal mammalian development and regulate genes involved in cell 
division and differentiation, metabolism, stress responses and apoptosis $(5,6)$. This post-transcriptional regulation appears to serve diverse and significant roles in multiple tissues of the female reproductive system (7).

Little is known about the function of miRNAs in human ULs. A number of studies have evaluated the levels of subclasses of miRNAs through microarray expression analysis $(8,9)$, demonstrating that numerous miRNAs are deregulated in leiomyomatous tissue compared with normal tissue. The majority of the studies assessing the effect of sex hormones on miRNA expression have been conducted via in vitro treatment of human cell lines with estradiol (E2). The first report on E2 regulation of miRNAs was in 2005, with the correlation between specific aberrant miRNA signatures, and $\mathrm{E}$ and $\mathrm{P} 4$ receptor status in breast cancer (10). Although there are numerous studies investigating in vitro the effect of ovarian sex steroids on growth factors, various cytokines, ECM-associated molecules, and cell proliferation and death in primary and immortalized leiomyoma cell lines, there are inadequate data on the regulation of miRNAs by E2 and P4 in the setting of intact ULs (2). The aim of the present study was to elucidate whether PGs affect UL growth compared with ovarian sex steroids via in vitro culture of leiomyoma and myometrial cells (LC and MC, respectively) obtained from hysterectomized patients.

\section{Materials and methods}

Chemicals and reagents. E2, celecoxib (Cele), P4, PGE2, mifepristone (MF), fulvestrant (ICI 182, 780) and PGF2 $\alpha$ were purchased from Sigma-Aldrich; Merck KGaA, (Darmstadt, Germany). Dulbecco's modified Eagle's medium (DMEM), fetal bovine serum (FBS), antibiotics-antimycotics and trypsin-EDTA were purchased from Invitrogen; Thermo Fisher Scientific, Inc., (Waltham, MA, USA). HBSS, collagenase and DNase were purchased from Invitrogen, and HEPES was purchased from Sigma-Aldrich; Merck KGaA.

Cell culture and treatment. UL tissues were obtained from patients undergoing hysterectomy after obtaining their written informed consent in accordance with the Ethics regulations of Dong-A University. The present study had been approved by the institutional review board of Dong-A University hospital in Busan, Republic of Korea. The present study included premenopausal women aged 30-50 years, who had not received any type of hormonal drug therapy to affect uterine function within at least 3 months prior to surgery. The tissues were minced and digested in collagenase solution (HEPES $25 \mathrm{mM}$, antibiotics $1 \mathrm{X}$, collagenase $2 \mathrm{mg} / \mathrm{ml}$ and DNase $0.2 \mathrm{mg} / \mathrm{ml}$ ) for $4 \mathrm{~h}$ at $37^{\circ} \mathrm{C}$ in a water bath. The digested tissues were passed through gauze to filter fragmented tissues, and the cells were collected by centrifugation and washed several times with PBS. The isolated cells in suspension were seeded in a $100 \mathrm{~cm}^{2}$ dish in culture medium (DMEM/F12) supplemented with 10\% FBS and 1X antibiotic-antimycotic solution at $37^{\circ} \mathrm{C}$ in a humidified atmosphere containing $5 \% \mathrm{CO}_{2}$ in air. The culture period was $72 \mathrm{~h}$ until measuring the miRNA levels. Cells were used in experiments between passages 4 and 8 . Drugs were added as 100X stock in DMSO or PBS. The culture duration and concentrations were determined through repetitive MTT assay.
The cell stock concentrations of E2, P4, PGE2 and PGF2 $\alpha$ were $1 \mu \mathrm{M}$. The concentrations of their antagonists, fulvestrant, MF and COX-2 inhibitor were $0.1 \mu \mathrm{M}$ in the culture media. Fulvestrant, MF and COX-2 inhibitor were administered together with E2, P4, PGE2 and PGF2 $\alpha$.

$R N A$ isolation and $R T-q P C R$. Total RNA was isolated using miRNeasy mini kit (Qiagen GmbH, Hilden, Germany), according to the manufacturer's protocol. cDNA was synthesized using a reverse transcription kit from Clontech Laboratories, Inc., (Mountainview, CA, USA) according to the manufacturer's protocol. qPCR was performed using SYBR green reagent (Qiagen $\mathrm{GmbH}$ ). Synthetic miRNA oligonucleotide primers were designed for the mature miRNAs, and purchased from Genolution Pharmaceuticals, Inc., (Song-pa, Seoul, South Korea). The details of the sequences are presented in Table II. The expression of miRNAs was normalized using U6 as an internal control. The Cq (quantification cycle) values were calculated from the amplification curve. The $2^{-\Delta \Delta C q}$ method was used to determine the relative quantification of miRNA expression. The miRNA experiment was performed under the following conditions: $95^{\circ} \mathrm{C}$ for $5 \mathrm{~min}$ as initial denaturation, followed by 40 cycles at $94^{\circ} \mathrm{C}$ for $15 \mathrm{sec}, 55^{\circ} \mathrm{C}$ for $34 \mathrm{sec}$, and $70^{\circ} \mathrm{C}$ for $30 \mathrm{sec}$.

Statistical analysis. The data are expressed as the mean \pm standard deviation for numerical values. Differences in study participants' characteristics were compared across subgroups with the analysis of variance with Tukey's post-hoc test or Kruskal-Wallis test with Dunn's post-hoc test, as appropriate. To determine if its distribution was normal, the present study used Shapiro-Wilk's test. $\mathrm{P}<0.05$ was considered to indicate a statistically significant difference. All statistical analyses were carried out using SPSS v.24.0 (IBM Corp., Armonk, NY, USA) statistical software.

\section{Results}

$P G$ effects on $L C$. The expression of four miRNAs (let-7a, miR-21, miR-26a and miR-200a) known to be involved in regulating cellular apoptosis was examined under treatment with sex steroids (E2 and P4) and PGs. PGE2 induced higher expressions of the three miRNAs (let 7a, miR26a and miR 200a), comparing with the NT or E2 significantly (Fig. 1). In particular, there were no statistical differences of expression in miR-21 group compared with other three miR groups (Fig. 1B). These findings indicated that PGs exerted a more potent anti-apoptotic effect on LC than E2. However, the expression patterns of PGF $2 \alpha$ differed from those of E2, except for miR-26a that PGF2 $\alpha$ induced as much as PGE2 (Fig. 1C). ICI did not reverse those effects induced by E2 (Fig. 1). MF and cele exhibited antagonistic action to P4 and PGE2 in only let-7a (Fig. 1A). Another four miRNAs (miR-29b, miR-93, miR-100 and miR-106b), known to regulate cellular inflammation, were examined under the same conditions. PGE2 did not induce the expressions of the four miRs as much as those of non-treated control or E2 significantly (Fig. 2). According to the mean expression fold ratio to E2, PGE2 exerted the most prominent anti-apoptotic and anti-inflammatory effects compared with other agents (Table I). 
Table I. miR fold ratios of $\mathrm{P} 4$, PGE2 and PGF2 $\alpha$ relative to $\mathrm{E} 2$ treatment.

\begin{tabular}{llcccc}
\hline & & \multicolumn{3}{c}{ miR fold ratio } \\
\cline { 4 - 5 } Variables & miRNAs & NT/E2 & P4/E2 & PGE2/E2 & PGF2 $\alpha /$ E2 \\
\hline LC (apoptosis-regulating miRNAs) & let-7a & 0.39 & 0.21 & 2.19 & 0.24 \\
& miR-21 & 0.38 & 1.41 & 3.83 & 0.39 \\
& miR-26a & 0.13 & 0.20 & 1.80 & 1.61 \\
& miR-200a & 0.25 & 0.45 & 2.08 & 0.51 \\
& Mean & 0.29 & 0.57 & 2.48 & 0.69 \\
LC (inflammation-regulating miRNAs) & miR-29b & 1.46 & 2.11 & 0.86 & 0.41 \\
& miR-93 & 2.02 & 1.17 & 1.10 & 0.82 \\
& miR-100 & 3.51 & 0.69 & 3.66 & 4.19 \\
& miR-106b & 1.22 & 0.78 & 0.38 & 0.23 \\
MC (apoptosis-regulating miRNAs) & Mean & 2.05 & 1.19 & 1.50 & 1.41 \\
& let-7a & 1.34 & 1.97 & 0.13 & 0.33 \\
& miR-21 & 2.22 & 22.34 & 10.29 & 6.27 \\
& miR-26a & 1.80 & 0.67 & 1.02 & 2.09 \\
MC (inflammation-regulating miRNAs) & miR-200a & 0.47 & 0.99 & 2.03 & 0.50 \\
& Mean & 1.46 & 6.49 & 3.37 & 2.30 \\
& miR-29b & 0.24 & 0.07 & 0.03 & 0.12 \\
& miR-93 & 0.26 & 0.24 & 0.24 & 0.01 \\
& miR-100 & 0.46 & 0.41 & 0.13 & 0.01 \\
& miR-106b & 0.61 & 0.43 & 2.43 & 3.09 \\
& Mean & 0.39 & 0.29 & 0.71 & 0.81 \\
\hline
\end{tabular}

NT, non-treated; E2, estradiol; ICI, fulvestrant; P4, progesterone; PG E2, prostaglandin E2; PGF2 $\alpha$, prostaglandin F2 $\alpha$; Cele, celecoxib; LC, leiomyoma cells; MF, mifepristone; MC, myometrial cells; miRNA/miR, microRNA.

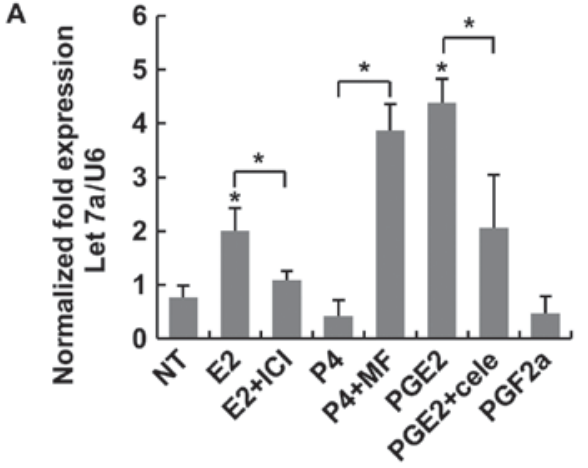

C

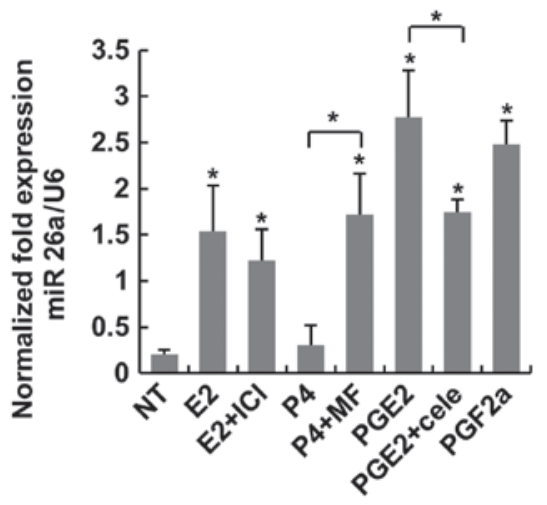

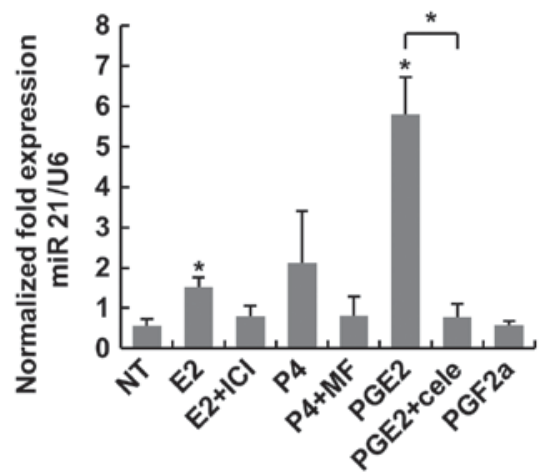

D

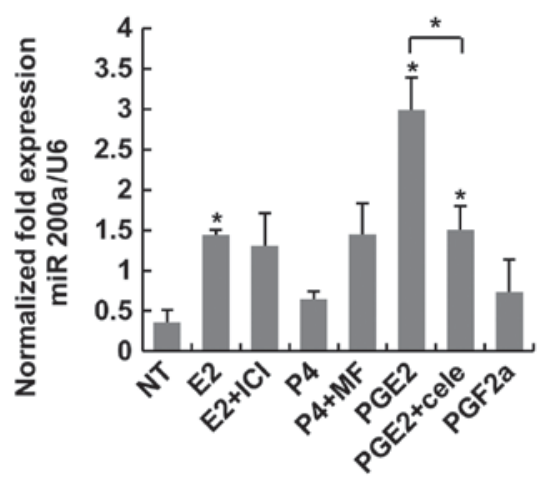

Figure 1. Expression of the miRNAs let-7a, miR-21, miR-26a and miR-200a was considered to regulate apoptosis in LC. (A) PGE2 significantly increased the expression of let-7a. (B) Statistical analysis of miR-21 across the groups. (C) PGE2 significantly increased the expression of miR 26a when compared with NT. (D) PGE2 significantly increased the expression of miR-200a when compared with NT. "P<0.05 vs. NT or indicated bracket. LC, leiomyoma cells; PG, prostaglandin; E2, estradiol; ICI, fulvestrant; P4, progesterone; NT, non-treated; MF, mifepristone; Cele, celecoxib. 
Table II. miR sequences known to be associated with anti-apoptosis and anti-inflammation in cultured myometrial and leiomyoma cells.

\begin{tabular}{llll}
\hline & Gene & Sequence (forward) & Accession no. \\
\hline Apoptosis-regulating miRNAs & let-7a-1 & TGAGGTAGTAGGTTGTATAGTT & MIMAT0000062 \\
& miR-21 & TAGCTTATCAGACTGATGTTGA & MIMAT0000076 \\
& miR-26a & TTCAAGTAATCCAGGATAGGCT & MIMAT0000082 \\
& miR-200a & TAACACTGTCTGGTAACGATGT & MIMAT0000682 \\
Inflammation-regulating miRNAs & miR-93 & CAAAGTGCTGTTCGTGCAGGTAG & MIMAT0000093 \\
& miR-106b & TAAAGTGCTGACAGTGCAGAT & MIMAT0000680 \\
& miR-100 & AACCGTAGATCCGAACTTGTG & MIMAT0000098 \\
& miR-29 & TAGCACCATTTGAAATCAGTGTT & MIMAT0000100
\end{tabular}

$\mathrm{miR} / \mathrm{miRNA}$, microRNA.
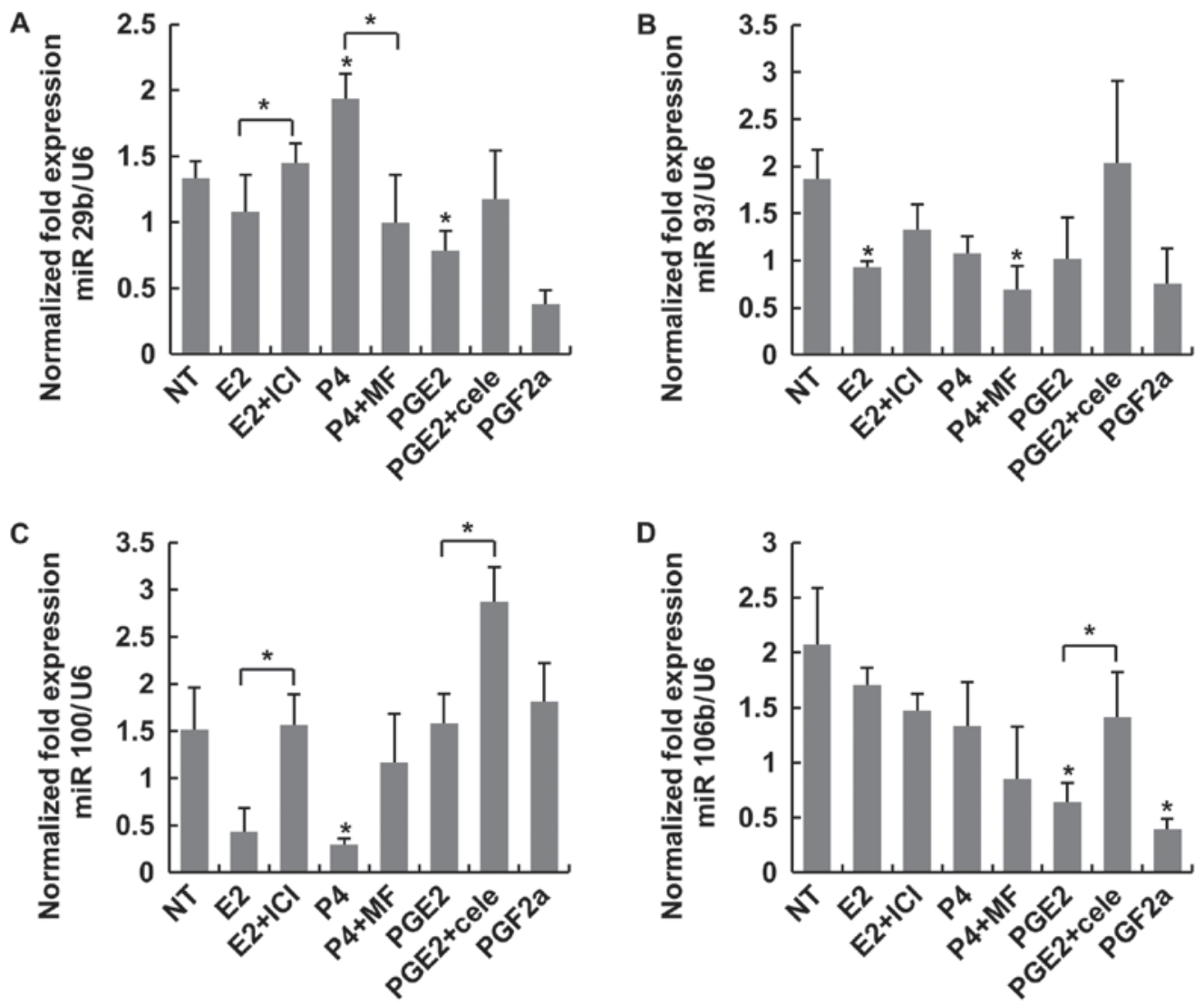

Figure 2. Expression of the miRNAs miR-29b, miR-93, miR-100 and miR-106b was considered to regulate inflammation in LC. (A) P4 induced a higher expression of miR-29b when compared with P4+MF. (B) No statistical differences were observed between treatment groups. (C) PGE2+cele induced a higher expression of miR-100 when compared with PGE2. (D) PGF2 $\alpha$ significantly decreased the expression of miR-106b when compared with NT. "P<0.05 vs. NT or indicated bracket. LC, leiomyoma cells; NT, non-treated; E2, estradiol; ICI, fulvestrant; P4, progesterone; MF, mifepristone; PG, prostaglandin; Cele, celecoxib.

$P G$ effects on MC. Overall, the miRNA expression patterns in MC differed compared with those in LC. Among the four miRNAs regulating $\mathrm{MC}$ apoptosis. PGE2 induced more expression of miR 200a compared with NT significantly (Fig. 3D). In other three miRs, there were no antagonist actions to sex-steroids or PGs. As regards miR-21, P4 induced higher expression compared with NT and E2 (Fig. 3B). As presented in Fig. 4, antagonists like MF or cele induced more expressions of miR
$29 \mathrm{~b}$ and miR 93 significantly. In terms of the expression ratios in MC, there was no significance among the miRs (Table I).

\section{Discussion}

Despite the high prevalence of ULs, their specific etiology remains largely unknown. Although the exact etiology of this transforming event is currently unknown, the neoplastic change 

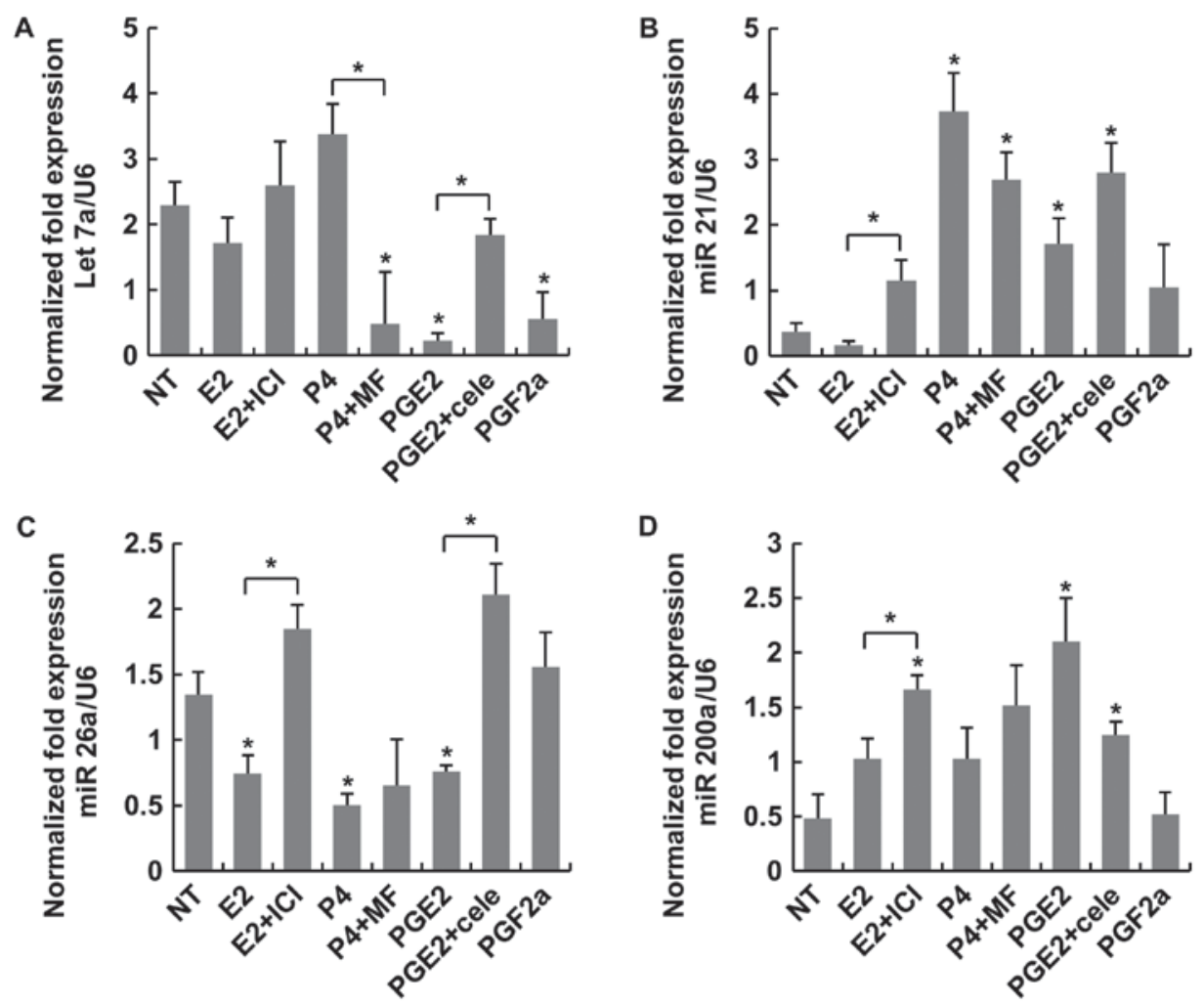

Figure 3. Expression of the miRNAs let-7a, miR-21, miR-26a and miR-200a was considered to regulate apoptosis in MC. (A) Statistical differences were not observed between PGE2 and E2. (B) P4 induced a higher expression of miR-21 than NT. (C) No statistical differences were observed between PGE2 and E2 for miR-26a expression. (D) PGE2 induced a higher expression of miR-200a when compared with NT. MC, myometrial cells; NT, non-treated; E2, estradiol; ICI, fulvestrant; P4, progesterone; MF, mifepristone; PG, prostaglandin; Cele, celecoxib. "P<0.05 vs. NT or indicated bracket.
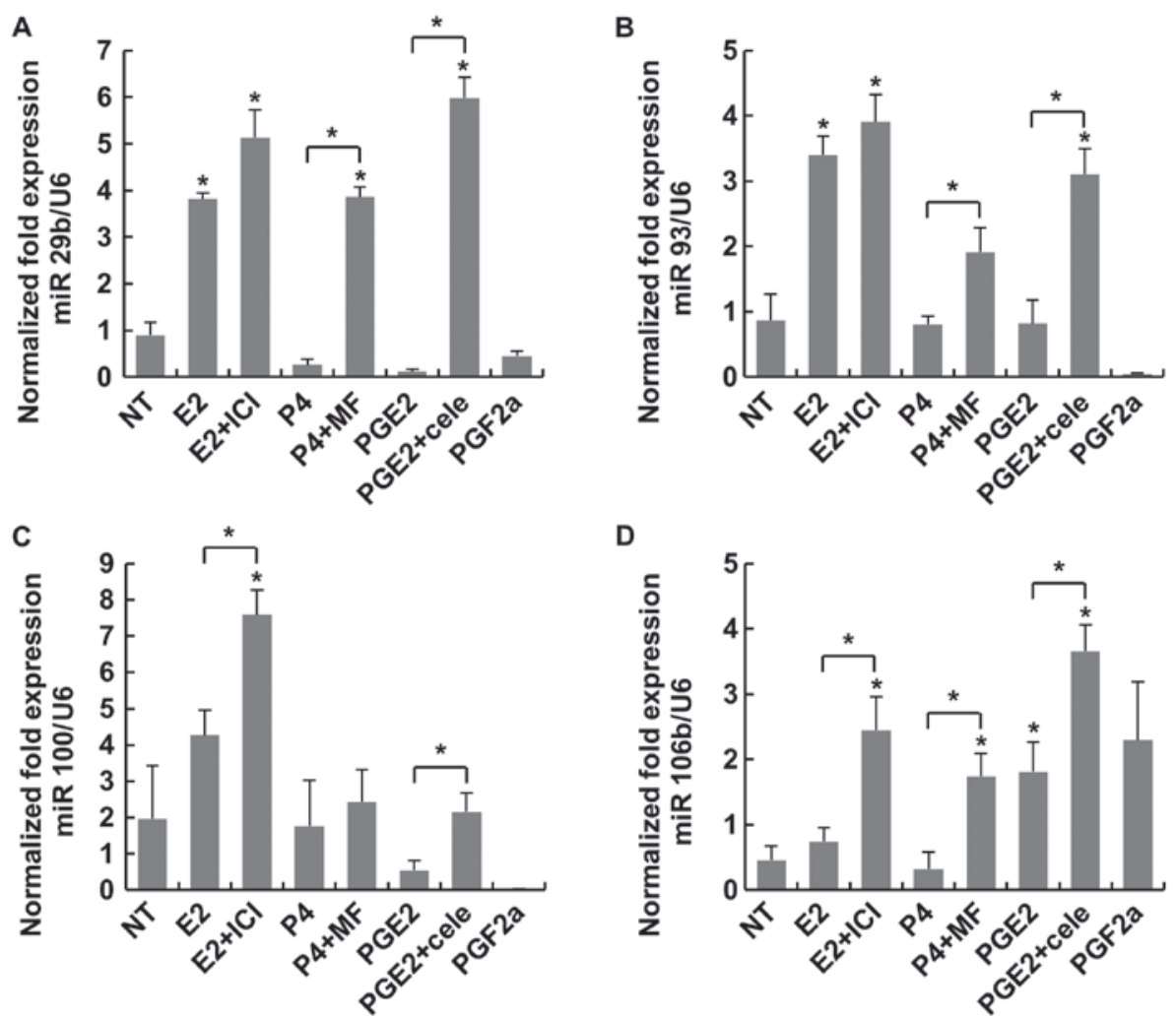

Figure 4. Expression of the miRNAs miR-29b, miR-93, miR-100 and miR-106b was considered to regulate inflammation in MC. (A) P4 plus MF and PGE2 plus cele induced significantly higher expression of miR-29b than P4 and PGE2 alone, respectively. (B) PGE2 plus cele induced a higher expression of miR-93 than NT and PGE2 alone. (C) There were no significant differences between treatment groups except for PGF2 $\alpha$. (D) PGE2 plus cele induced a higher expression when compared with NT. "P<0.05 vs. NT or indicated bracket. MC, myometrial cells; NT, non-treated; E2, estradiol; ICI, fulvestrant; P4, progesterone; MF, mifepristone; PG, prostaglandin; Cele, celecoxib. 
of a myometrial cell is likely to be due to a cellular insult (11). Regardless of the cellular insult, a common primary characteristic of ULs is their responsiveness to steroid hormones. E2 and P4 lead to tumor growth by stimulating a modest rate of cell proliferation and the production of abundant amounts of extracellular collagen matrix.

There is a 3-fold increase in PG levels in the endometrium from the follicular to the luteal phase, with a further increase during menstruation (12). Most of the PG release during menstruation occurs during the first $48 \mathrm{~h}$, which corresponds to the greatest intensity of the symptoms. However, the effects of PGs on UL growth have not been adequately investigated to date. In this study, we found that PGs may promote the growth of cultured LC as a result of the miRNA expression fold ratio to E2. However, further in vivo studies are required to validate the effects of PGs in the future.

Since the introduction of miRNAs, accumulating evidence of abnormal miRNA expression has revealed their function in normal biological activities, and has provided a novel insight into their potential functional significance in a wide range of common human diseases, such as malignancy, cardiac disease, diabetes and insulin resistance (13-15). Increased expression of a specific miRNA causes the suppression of translation of the targeted mRNA, whereas downregulation of the miRNA exerts the opposite effect. These translational modification processes induce distinct protein expression profiles, causing various cellular and tissue changes. The functional significance of differential miRNA expression under normal and disease conditions is not always as apparent as may be expected. We herein investigated eight miRNAs known to regulate the cellular events of apoptosis or inflammation, based on previous studies $(2,16,17)$. Dysregulated expression of miRNAs such as let-7, miR-21, miR-92, miR-106b and miR-200 has been reported to be associated with the development of leiomyomas (17). In this study, we tried to evaluate the potential biologic effects of PGs from menstruating uterus through expression ratios of the eight miRNAs comparing with those of sex steroid hormones.

The expression patterns of miRNAs in this experiment were not consistent with previous studies. For example, in a previous study, it was reported that E2 reduced the expression of miR-21 and miR-26a, and the pattern was reversed by treatment with the anti-estrogen compound fulvestrant in MC and LC (9). However, co-treatment with E2 and fulvestrant decreased the expression of miR-21 and miR-26a in the present experiment. These opposite results may be attributed to the fact that the present study was performed under different in vitro culture conditions. Moreover, although $>1,000$ miRNAs have been identified in humans recently, their detailed functional role in gene regulation has not been adequately investigated thus far. The intracellular anti-apoptotic or anti-inflammatory functional roles of the eight miRNAs examined in the present study were based on previous studies. It is considered that such a functional analysis of miRNAs may be helpful in understanding the role of miRNAs, as the effects of $\mathrm{E}$ and $\mathrm{P} 4$ on the cell are well known. Furthermore, as translational research into the application of miRNAs is progressing, diagnostic and therapeutic biomarkers may have the potential to change the standard of care of ULs.

In conclusion, PGE2, a key inflammatory mediator, which is produced in copious amounts during menstruation, may be a potential promoter of UL growth. Therefore, reducing the amount or antagonizing the action of PGE2 produced by the menstruating uterus may be considered as an alternative therapeutic strategy.

\section{Acknowledgements}

Not applicable.

\section{Funding}

This study was supported by Research Institute for Convergence of Biomedical Science and Technology (30-2016-000), Pusan National University Yangsan Hospital.

\section{Availability of data and materials}

The datasets used and/or analyzed during the current study are available from the corresponding author on reasonable request.

\section{Authors' contributions}

HK and YC conceived and designed the experiments. GJ and SP performed cell culture, RNA isolation and RT-qPCR. YC and $\mathrm{MH}$ recruited the patients who had provided their extirpated uterus for this study. MH analyzed the data. All authors read and approved the final manuscript.

\section{Ethics approval and consent to participate}

Written informed consent was obtained from all patients (age range, 40-50) at the Department of Obstetrics and Gynecology, Dong-A University Hospital (Busan, Republic of Korea) that participated in the study, and the study was approved by the Ethics Committee of Dong-A University.

\section{Patient consent for publication}

Written informed consent was obtained from all patients.

\section{Competing interests}

The authors declare that they have no competing interests.

\section{References}

1. Myers ER, Barber MD, Gustilo-Ashby T, Couchman G, Matchar DB and McCrory DC: Management of uterine leiomyomata: What do we really know? Obstet Gynecol 100: 8-17, 2002.

2. Karmon AE, Cardozo ER, Rueda BR and Styer AK: MicroRNAs in the development and pathobiology of uterine leiomyomata: Does evidence support future strategies for clinical intervention? Hum Reprod Update 20: 670-687, 2014.

3. Park SB, Jee BC, Kim SH, Cho YJ and Han M: Cyclooxygenase-2 inhibitor, celecoxib, inhibits leiomyoma cell proliferation through the nuclear factor $\kappa$ B pathway. Reprod Sci 21: 1187-1195, 2014.

4. Bartel DP: MicroRNAs: Target recognition and regulatory functions. Cell 136: 215-233, 2009.

5. Alvarez-Garcia I and Miska EA: MicroRNA functions in animal development and human disease. Development 132: 4653-4662, 2005.

6. Georgieva B, Milev I, Minkov I, Dimitrova I, Bradford AP and Baev V: Characterization of the uterine leiomyoma microRNAome by deep sequencing. Genomics 99: 275-281, 2012. 
7. Carletti MZ and Christenson LK: MicroRNA in the ovary and female reproductive tract. J Anim Sci 87 (14 Suppl): E29-E38, 2009.

8. Marsh EE, Lin Z, Yin P, Milad M, Chakravarti D and Bulun SE: Differential expression of microRNA species in human uterine leiomyoma versus normal myometrium. Fertil Steril 89: 1771-1776, 2008.

9. Pan Q, Luo X and Chegini N: Differential expression of microRNAs in myometrium and leiomyomas and regulation by ovarian steroids. J Cell Mol Med 12: 227-240, 2008

10. Iorio MV, Ferracin M, Liu CG, Veronese A, Spizzo R, Sabbioni S, Magri E, Pedriali M, Fabbri M, Campiglio M, et al: MicroRNA gene expression deregulation in human breast cancer. Cancer Res 65: 7065-7070, 2005.

11. Moravek MB, Yin P, Ono M, Coon JS V, Dyson MT, Navarro A, Marsh EE, Chakravarti D, Kim JJ, Wei JJ and Bulun SE: Ovarian steroids, stem cells and uterine leiomyoma: Therapeutic implications. Hum Reprod Update 21: 1-12, 2015.

12. Eldering JA, Nay MG, Hoberg LM, Longcope C and McCracken JA: Hormonal regulation of prostaglandin production by rhesus monkey endometrium. J Clin Endocrinol Metab 71: 596-604, 1990.
13. Wu H, Xiao Z, Wang K, Liu W and Hao Q: MiR-145 is downregulated in human ovarian cancer and modulates cell growth and invasion by targeting p70S6K1 and MUC1. Biochem Biophys Res Commun 441: 693-700, 2013.

14. Thum T and Mayr M: Review focus on the role of microRNA in cardiovascular biology and disease. Cardiovasc Res 93: 543-544, 2012.

15. Zhu H,Shyh-Chang N,SegrèAV,Shinoda G,Shah SP,Einhorn WS, Takeuchi A, Engreitz JM, Hagan JP, Kharas MG, et al: The Lin28/let-7 axis regulates glucose metabolism. Cell 147: 81-94, 2011.

16. Chuang TD, Luo X, Panda H and Chegini N: miR-93/106b and their host gene, MCM7, are differentially expressed in leiomyomas and functionally target F3 and IL-8. Mol Endocrinol 26: 1028-1042, 2012

17. Chuang TD, Panda H, Luo $X$ and Chegini N: miR-200c is aberrantly expressed in leiomyomas in an ethnic-dependent manner and targets ZEBs, VEGFA, TIMP2, and FBLN5. Endocr Relat Cancer 19: 541-556, 2012. 